\title{
Overwhelming pulmonary infection after a tobogganing accident
}

\author{
Ravinder Singh MD FRCSC ${ }^{1}$, Brian E Louie MD FRCSC ${ }^{1}$, William F Bennett MD FRCSC ${ }^{1}$, \\ Christopher Allen MD FRCPC ${ }^{2}$, Tom Kelly MLT ART ${ }^{3}$, Christine H Lee MD FRCPC ${ }^{3}$
}

\section{CASE PRESENTATION}

A 17-year-old male patient presented to St Joseph's Healthcare (Hamilton, Ontario) with a radiologically opacified left hemithorax. Three days earlier, the patient had injured his left lower chest while tobogganing on his farm. He developed dyspnea and felt unwell, but only sought medical attention from his family doctor a few days after the injury, when fever and pleuritic chest pain ensued. He was treated with a nonsteroidal anti-inflammatory agent, but his chest radiograph revealed an opacified hemithorax, for which he was referred to the hospital. In the emergency department, the patient looked ill and was in distress. His heart rate was 125 beats/min, and he had a blood pressure of $103 / 61 \mathrm{mmHg}$, a respiratory rate of 20 breaths $/ \mathrm{min}$, a temperature of $38.5^{\circ} \mathrm{C}$ and an oxygen saturation of $94 \%$ on ambient air. Laboratory results showed a white blood cell count of $40 \times 10^{9} / \mathrm{L}$ with a left shift. Chest radiography showed a left pleural effusion. A \#28 Fr chest tube was inserted into the left hemithorax, and foul-smelling serosanguineous fluid was drained. There was a transient improvement of tachypnea and hypoxemia despite minimal radiographic change. He was admitted and subsequently started on intravenous levofloxacin. Overnight, he deteriorated and required an increase in supplemental oxygen. A computed tomography (CT) scan of his chest revealed multiple loculated fluid collections and bilateral pulmonary parenchymal infiltrates consistent with a pneumonia and empyema.

He was urgently taken to the operating room for open drainage and decortication of the left chest empyema. At thoracotomy, the pleura were edematous, hyperemic and thickened with loculated collections of pus anteriorly and superiorly over the left upper lobe. There was inflammatory hypertrophy of the pericardial fat. The lingula was hyperemic and firm. The rest of the lung appeared healthy. Enucleation of the empyema and decortication of the pleura were both undertaken. The pleural space was lavaged with povidone-iodine followed by normal saline. The chest was closed over three tube thoracostomies. Bronchoscopy, for pulmonary toilet, revealed copious amounts of blood-stained secretions. The patient was transferred to the
\end{abstract}

intensive care unit for general medical care, inotropic support and mechanical ventilation. Intravenous piperacillin-tazobactam was administered and the chest tube was maintained for ongoing drainage.

The Gram stain of the pleural fluid collected before the initiation of any antibiotic showed a moderate amount of pus cells and Gram-negative bacilli (Figure 1). No organism grew aerobically but the anaerobic incubation produced the growth of a single type of Gram-negative bacillus. Postoperatively, the patient exhibited slow but steady improvement. As the septic process subsided, the patient was weaned from both inotropic and ventilatory supports. The only organism isolated from the empyema was the Gramnegative anaerobic bacillus.

Serial CT scans of the patient's chest showed significant resolution of the intrathoracic and pulmonary processes. The chest tubes were removed before discharge. Antibiotic therapy with piperacillin-tazobactam was continued for four weeks. The patient was discharged to his home. At one-month follow-up, he was ambulating and doing well. What anaerobic isolate grew from the pleural fluid?

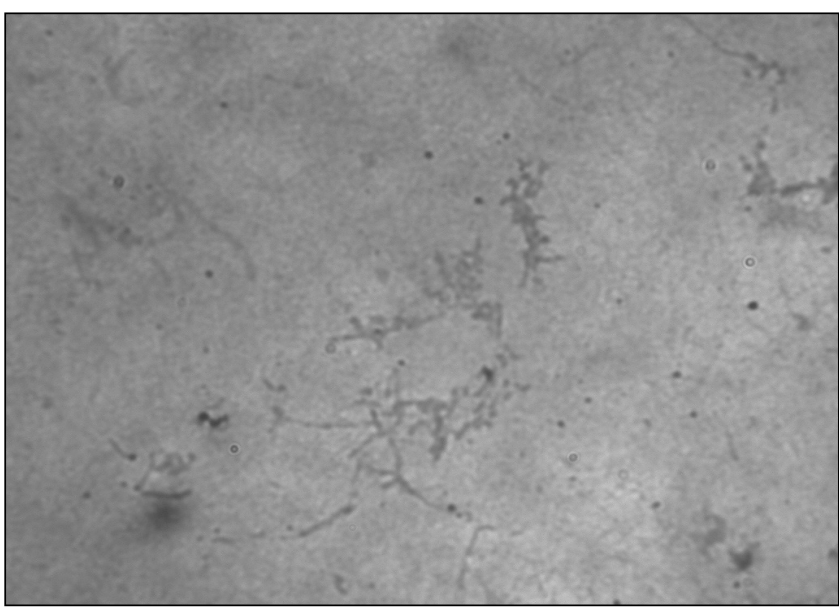

Figure 1) Gram stain of the patient's pleural fluid

${ }^{1}$ Department of Surgery; ${ }^{2}$ Department of Medicine; ${ }^{3}$ Hamilton Regional Laboratory Medicine Program, McMaster University, Hamilton, Ontario Correspondence: Dr Christine Lee, Medical Microbiology, Infectious Diseases, St Joseph's Healthcare, 424 Luke Wing, 50 Charlton Avenue East,

Hamilton, Ontario L8N 4A6. Telephone 905-521-6021, fax 905-521-6083, e-mail clee@mcmaster.ca

Received and accepted for publication October 13, 2004 


\section{DIAGNOSIS}

The Gram stain of the pleural fluid collected from the patient before the initiation of any antibiotic showed a moderate amount of pus cells and pale staining, pleomorphic Gramnegative bacilli. No organism grew aerobically. After $48 \mathrm{~h}$ of anaerobic incubation on Brucella and Neomycin anaerobic agar, a characteristic colonial morphology of Fusobacterium necrophorum was present in the form of small, grey, translucent colonies with greening of the sheep blood in the agar. Gas liquid chromatography analysis of this isolate growing in prereduced anaerobically sterilized chopped meat glucose broth showed the presence of acetic, propionic and butyric acids, all of which are volatile, short-chain fatty acids. The increased production of propionic acid in culture is diagnostic for $F$ necrophorum. The speciation of the organism was further obtained by the RapID ANA II System (Remel Inc, USA), biotype code 000104, and the production of lipase on egg yolk agar.

\section{DISCUSSION}

F necrophorum is a Gram-negative anaerobic bacillus that is normally found in the oral and upper respiratory tract of humans. It can also be found in the large bowel, terminal ileum and female genital tract. It is a major pathogen in farm animals. Infection requires inoculation of an existing nidus with F necrophorum and interaction with its endotoxin and exotoxin (leukotoxin) $(1,2)$. Courmont and Cadde first reported infection by this organism in 1900. Lemierre, in a review of 20 cases $(1,3)$, reported a mortality rate of $90 \%$. Lemierre (1) set the criteria for the diagnosis of this infection (which was later known as Lemierre syndrome or post-anginal septicemia): previous oropharyngeal infection; septicemia after four or five days; swelling and unilateral tenderness around the angle of the jaw or parallel to the sternocleidomastoid muscle; metastatic abscesses; isolation of $F$ necrophorum from blood or abscess cavity; and rapid progression. Oropharyngeal infection can lead to septic thrombosis of the internal jugular vein, which can embolize to distant sites, and the lungs are the most common site (79.8\%) (2-4).

There have been 116 cases of Lemierre syndrome in the literature, but its occurrence has decreased over time because of prophylactic antibiotic use before oropharyngeal procedures and better oral hygiene. Lemierre syndrome is also likely underreported because of the fastidious nature of the organism. $F$ necrophorum is difficult to grow and sustain in culture because it is highly sensitive to oxygen (5). As well, its cellular morphology is not typical of the genus because it is pleomorphic rather than needle-like or fusiform.

In the present case, the patient reported symptoms of an upper respiratory tract infection consisting of a sore throat, fever, cough and headache that resolved before his trauma. His symptoms rapidly progressed after the trauma, and he developed septic shock within three days. An ultrasound Doppler of his carotid arteries and jugular veins was found to be normal. Also, the patient did not have any evidence of swelling or tenderness around the angle of the jaw or parallel to the sternocleidomastoid muscle. Thus, although he did not meet all of the criteria for Lemierre syndrome, not all of these signs may have been evident at the time of presentation. We believe that our patient's clinical course was due to microaspiration. Direct inoculation is not a plausible etiological mechanism in this patient because injection of the $F$ necrophorum from his farm into his left hemithorax would require either a puncture or an open fracture. His chest trauma was minor, with no clinical or radiological evidence of a puncture or rib fractures. A more plausible theory is that of microaspiration. During the trauma, the patient may have aspirated oropharyngeal contents while sustaining a very mild pulmonary contusion. We theorize that inoculation of the contusion led to a pneumonic process including parapneumonic effusion that subsequently became infected.

Drainage of pus and debridement of necrotic tissue are essential components of managing anaerobic infections. This is also the case for the treatment of any type of empyema. Levofloxacin does not have reliable antimicrobial activity against Fusobacterium species. Most Fusobacterium species remain susceptible to penicillin, although rare instances of treatment failure have been reported due to the production of beta-lactamase $(6,7)$.

\section{REFERENCES}

1. Briggs S, Pappachan J, Argent J, McGill N, Marsh M. Lemierre disease in the pediatric intensive care unit, clinical course, and the use of high-frequency oscillatory ventilation. Pediatr Crit Care Med 2003;4:107-10.

2. Hoehn S, Dominguez TE. Lemierre's syndrome: An unusual cause of sepsis and abdominal pain. Crit Care Med 2002;30:1644-7.

3. Jaremko JL, Kirton A, Brenner JL. A 12-year-old girl with pharyngitis, meningitis and sinovenous thrombosis. CMAJ 2003;169:811-2.

4. Chirinos JA, Lichtstein DM, Garcia J, Tamariz LJ. The evolution of Lemierre syndrome: Report of 2 cases and review of the literature. Medicine 2002;81:458-65.

5. Fiesseler FW, Richman PB, Riggs RL. Pharyngitis followed by hypoxia and sepsis: Lemierre syndrome. Am J Emerg Med 2001;19:320-2.

6. Brook I, Yocum P, Foote PA Jr. Changes in the core tonsillar bacteriology of recurrent tonsillitis: 1977-1993. Clin Infect Dis 1995;21:171-6.

7. Goldstein EJ, Summanen PH, Citron DM, Rosove MH, Finegold SM. Fatal sepsis due to a beta-lactamase-producing strain of Fusobacterium nucleatum subspecies polymorphum. Clin Infect Dis 1995;20:797-800. 


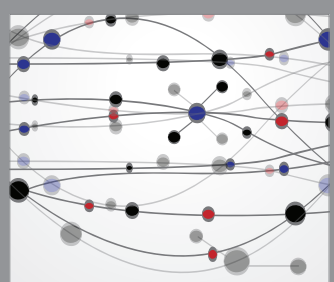

The Scientific World Journal
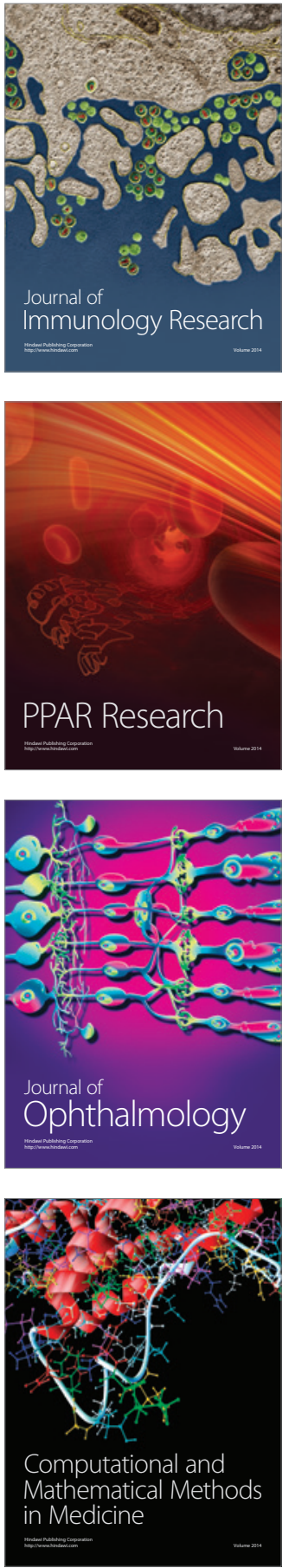

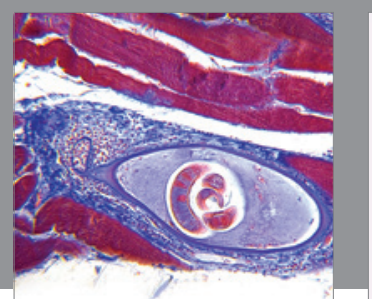

Gastroenterology Research and Practice

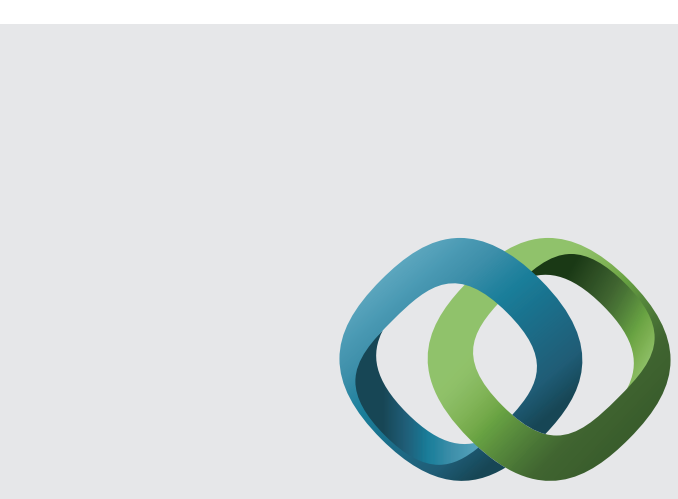

\section{Hindawi}

Submit your manuscripts at

http://www.hindawi.com
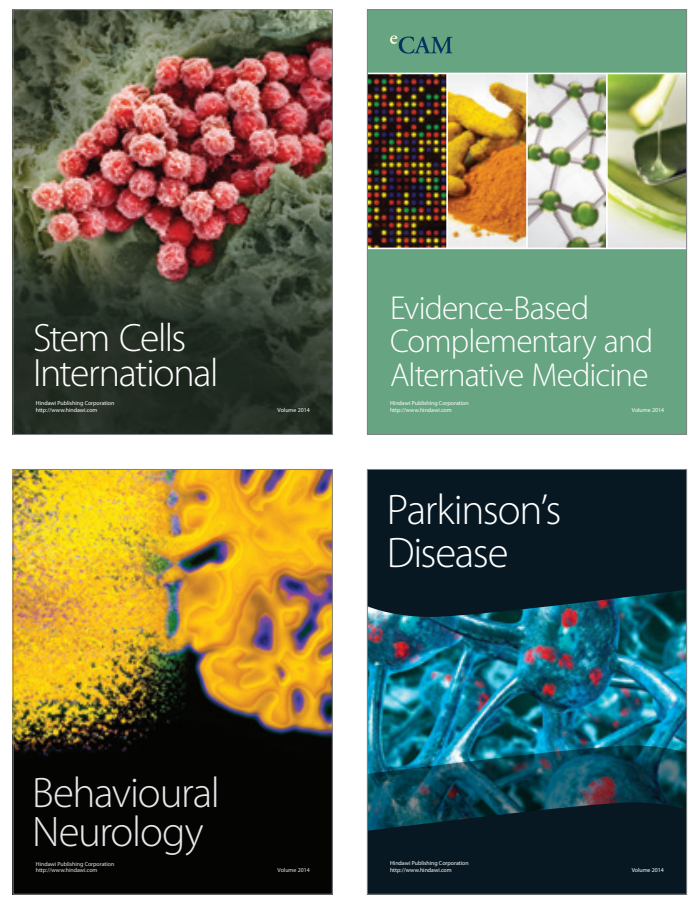
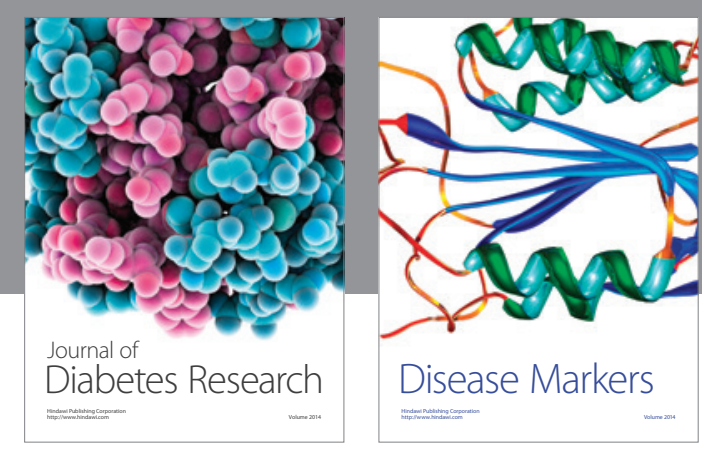

Disease Markers
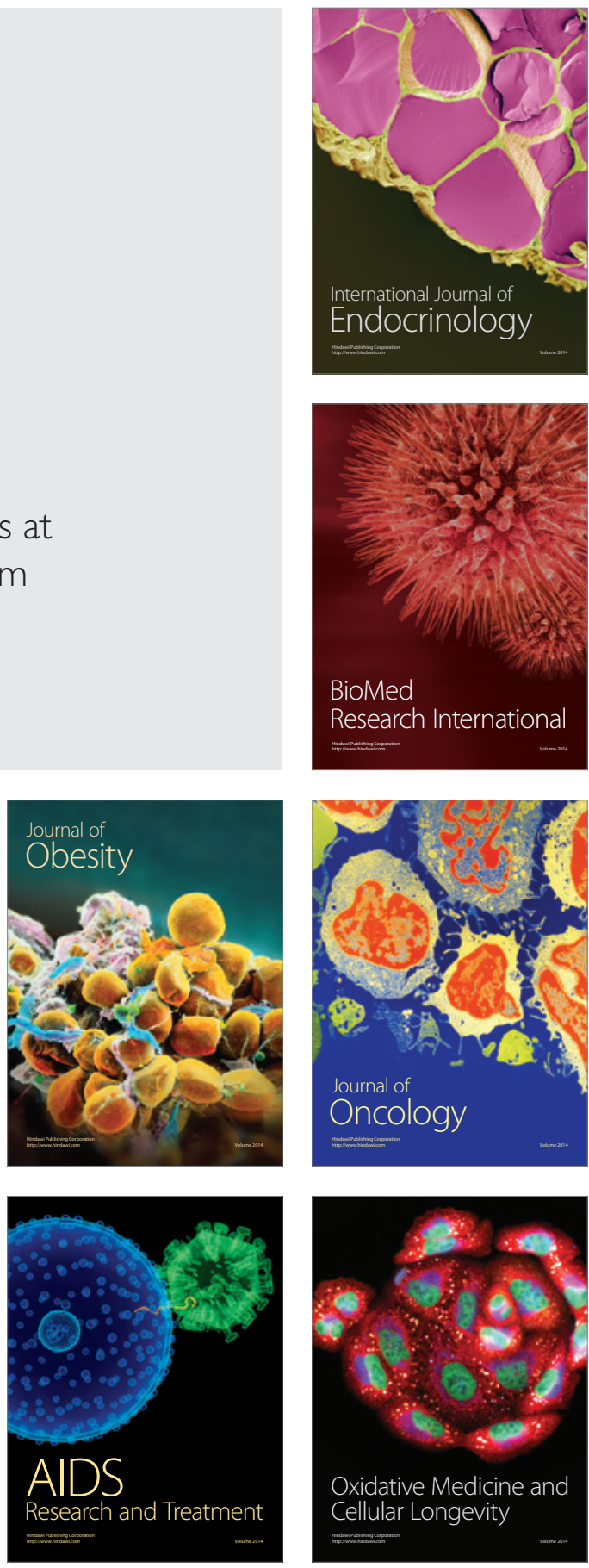